\title{
Robust Optimum Trajectory Design of a Satellite Launch Vehicle in the Presence of Uncertainties
}

\author{
Reza Zardashti1,*, Mahdi Jafari¹, Sayyed Majid Hosseini ${ }^{1}$ Sayyed Ali Saadatdar Arani
}

Reza Zardashti (iD https://orcid.org/0000-0003-0490-3584

Mahdi Jaffari (iD https: / / orcid.org/0000-0003-1760-5877

Sayyed Majid Hosseini (iD https://orcid. org/0000-0003-1277-1104

Sayyed Ali Saadatdar Arani (iD https: / / orcid. org/0000-0002-6886-3158

\section{How to cite}

Zardashti R, Jafari M, Hosseini SM, Arani SAS (2020) Robust Optimum Trajectory Design of a Satellite Launch Vehicle in the Presence of Uncertainties. J Aerosp Tecnol Manag, 12 e3520. https://doi. org/10.5028/jatm.v12.1176

\begin{abstract}
In this paper, a robust optimization method is developed to solve the Satellite Launch Vehicle (SLV) trajectory design problem in the presence of uncertainties. Given these uncertainties in the actual SLV ascent trajectory, it is important to find an optimal trajectory that is resistant to these uncertainties, as it results in increased flight performance, reduced steering-control system workload and increased SLV reliability. For this purpose, the optimization problem is first considered by applying to maximize the payload mass criterion as an objective function and three-dimensional equations of motions as the governing constraints. Then by adding mean and standard deviation parameters of uncertainties, the robust optimizer model is developed and the genetic algorithm is used to execute the model. Monte Carlo simulation is also used to analyze the results of uncertainties and its continuous feedback to the optimizer model. Finally, an optimal trajectory is obtained that is robust to the uncertainties effects such as aerodynamic coefficients, dry mass and thrust errors of the SLV. The results of the simulation show the validity of this claim.
\end{abstract}

KEYWORDS: Optimum robust; Genetic algorithm; Trajectory Design; Monte Carlo Simulation.

\section{INTRODUCTION}

The optimization of a system is achieved by minimization or maximization of an objective function characterized by the system performance. This will ultimately improve the system performance. Aerospace systems are so large and complex in their practical dimensions that their optimal solution cannot be achieved in an acceptable amount of time. Therefore, it is necessary to be satisfied with the near-optimal solutions so that they have acceptable quality and are obtained in an acceptable time (Wazed, 2009).

The optimization of real systems is achieved according to different criteria. One of the most important of these criteria is uncertainty. Uncertainty is an integral part of real dynamic systems. In many cases, it is not possible to accurately predict the parameters and factors affecting the system, and at best there is only an estimate of them. In many cases, ignoring these uncertainties can increase the systems overall cost, and in some cases even undermine the value of the solutions offered (Yao et al. 2011).

The main reason for the uncertainty is that "insufficient knowledge (both in information and in concepts) makes the predicted model different from the real one". Based on this, the uncertainty in aerospace systems can be divided into "input", "modeling",

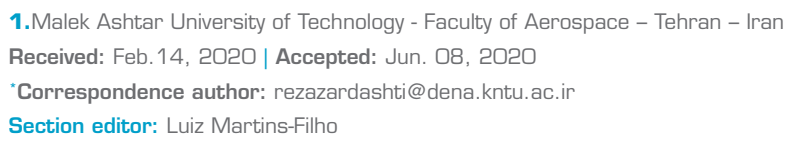


"measurement" and "operational / environmental" uncertainties, respectively (Fig. 1). Uncertainty in "input" occurs when the requirements for determining a design problem are inaccurate, ambiguous, or undefined. The uncertainty of "model parameters" is related to the error in all mathematical models used to describe physical systems. The uncertainty of "measurement" occurs when the desired output is not fully calculated through a mathematical model. Ultimately, "operational / environmental" uncertainty is the result of unknown / uncontrollable external disturbances.

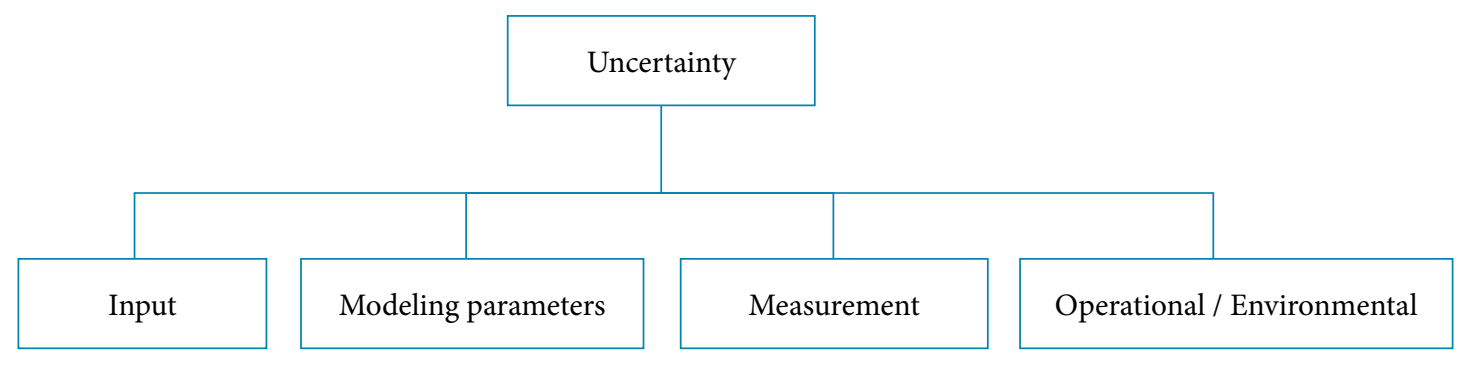

Figure 1. Uncertainty classification in systems analysis and design.

The Trajectory Design of a real SLV is a complex problem, so it needs devoted methodologies to solve the problem. In this regard, two major classes of the uncertainty-based optimization are developed: robust optimization and reliabilitybased optimization. The first method is formulated based on the mean value and standard deviation parameters of the normal distribution of uncertainties, while in the second method, the reliability values of the system are used as the optimization criterion.

Koch et al. (2000) and Marvis \& Delaurentis (2000) were published articles on the robust optimization problem. Sues and Cesare (2000) raised the question of reliability in design. According Akhtar and Linshu (2006), researchers performed robust optimization of a multi-stage satellite and supplemented their previous work with that of other researchers in the field of integrating MDO with robust design and introduced a method called Modified Concurrent Subsystem Uncertainty Analysis (MCSSUA) that improved previous work, namely, Concurrent Subsystem Uncertainty Analysis (CUSSA). Also, in the continuation of this work, the robust design entered the participatory optimization structure and then a hierarchical structure was invented, which included three levels of system, subsystem, and uncertainty analysis. They report that although this method is effective, some problems are encountered due to the problem of convergence of collaborative optimization and sensitivity to the starting point in subsystem optimization.

Zaman et al. (2009) presented the design optimization based on uncertainty resistance in Information and proposes formulation and algorithmic design optimization under both uncertainties, chance (i.e., physical or natural variability), and cognitive (i.e., inaccurate probability information), from the standpoint of system resistance. Yao et al. (2011) present a complete overview of all the work done to date, with a review of uncertainty design optimization methods for aerospace devices presented. Bataleblu et al. (2011) presented the optimization of robust design of liquid fuel launch vehicle with uncertainty, in which they expressed the multiobjective optimization problem with simplified optimization and simulation methods. To reduce the mass of the launch vehicle in the presence of the uncertainty has been addressed. Liu and $\mathrm{Lu}$ (2013) presented a formulation and algorithm for generating an optimal finite trajectory for meeting in an arbitrary orbit.

Paiva et al. (2014) presented a model for simultaneous analysis, reliability, and calculation of aircraft reliability. Okada et al. (2015) presented a robust trajectory design for a projectile in which the effectiveness of the proposed method shown by using the Monte Carlo simulation. Su and Wang (2015) expressed a robust optimized approach for the reentry phase of a reusable launch vehicle trajectory.

Luo and Yang (2017) investigated the orbital uncertainty propagation, using Monte Carlo and some linear models simulation. Grant (2015) provides a robust trajectory design using indirect optimization methods, in which it captures the worst-case scenario for each scattering of the model and reduces the dimensions of the design problem without the need to publish statistical 


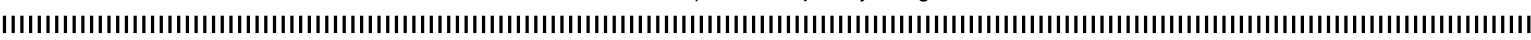

information. Xue and Duan (2017) have published the attitude robust control for a reusable launch vehicle in the reentry phase based on the fractional calculus and Pigeon-inspired optimization.

In this paper, the optimization problem is first considered by applying the payload mass maximization criterion as a cost function and three-dimensional equations of motions as the constraint equations. Then by adding mean and standard deviation parameters of some uncertainties such as the SLV aerodynamic coefficients, dry mass and engine thrust, the robust optimizer model is developed. In the following, a genetic algorithm (GA) is used as solver to execute the optimization model. Monte Carlo simulation is also used to analyze the results of uncertainties and feedback the information to the optimizer model. For this purpose, the rest of the paper is organized as follows. In the next section, the SLV mathematical modeling is presented and subsequently, the optimization formulation is also presented in two cases of deterministic optimal and robust optimal formulation. The simulation results are presented in a later section, and the last section completes the paper with a conclusion.

\section{THE SLV MATHEMATICAL MODELING}

\section{THREE-DIMENSIONAL EQUATIONS OF MOTIONS}

The dynamic model uses a 3 DOF translational equations of motion for a variable-mass point in the rotating Earth-centered Earth-fixed (ECEF) reference frame. These equations are given for the translational position $[h ; \tau ; \lambda]^{T}$ and velocity $[V ; \dot{\gamma} ; \dot{\Psi}]^{T}$ vectors in the spherical coordinates (Vinh, 1981):

$$
\begin{aligned}
& \dot{\lambda}=\frac{V \cos \gamma \cos \psi}{r \cos \tau} \\
& \dot{\tau}=\frac{V \cos \gamma \cos \psi}{r} \\
& \dot{h}=V \sin \gamma \\
& \dot{V}=\frac{1}{m}(T \cos (\alpha+\delta)-D-m g \sin \gamma)+r \omega^{2} \cos \tau(\cos \tau \sin \gamma-\sin \tau \cos \gamma \sin \psi) \\
& \dot{\gamma}=\frac{1}{m V}[(T \sin (\alpha+\delta)+L) \cos \mu-m g \cos \gamma]+\frac{V \cos \gamma}{r}+2 \omega \cos \tau \cos \psi+\frac{r \omega^{2}}{V} \cos \tau(\cos \tau \cos \gamma+\sin \tau \sin \gamma \sin \psi) \\
& \dot{\psi}=-\frac{1}{m V \cos \gamma}(T \sin (\alpha+\delta)+L) \sin \mu-\frac{V}{r} \tan \tau \cos \gamma \cos \psi+2 \omega(\cos \tau \tan \gamma \sin \psi-\sin \tau)-\frac{r \omega^{2}}{V \cos \gamma}(\cos \tau \sin \tau \cos \psi) \\
& \dot{m}=-\frac{1}{I_{s p} g_{0}} T_{v a c}
\end{aligned}
$$

In the above equations, $l$ is the longitude, $\tau$ is the latitude, $h$ is the height above mean sea level, $V$ is the total velocity, $g$ is the flight path angle, $\psi$ is the heading angle, $\mathrm{m}$ is the mass, $r$ is the distance from the center of the Earth to the vehicle mass center, $w$ is the angular velocity of the earth, $D$ is the drag, $L$ is the lift, $T$ is the thrust, $I_{s p}$ is the specific impulse, $d$ is the gimbal angle of the thrust vector, $a$ is the angle of attack, and $m$ is the velocity roll angle (take zero in this manuscript). The Fig. 2 illustrates schematically parameters of the motion. 


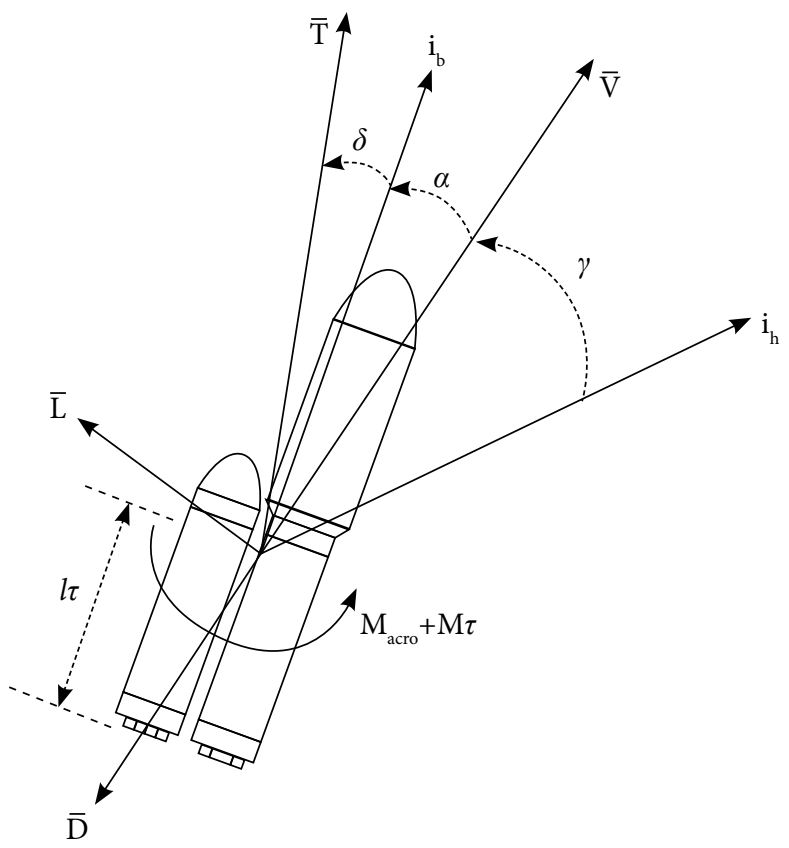

Figure 2. launch vehicle force's and moment's nomenclature [Hesami Rostami and Tolouie, 2015]

\section{PITCHING PROGRAM OF THE SLV TRAJECTORY}

Because the SLV motion is a guided motion, the pitch angle as a control function must be specified to satisfy flight constraints. These constraints include reaching orbital velocity and height while the trajectory angle is zero at the moment of orbital injection. Here, the pitch angle is considered for the first step of a quadratic form while the second step of the linear form according to the following equation:

$$
\theta(t)= \begin{cases}\frac{\pi}{2} & 0 \leq t \leq t_{0} \\ \frac{\frac{\pi}{2}-\theta_{1}}{\left(t_{1}-t_{0}\right)^{2}}\left(t_{1}-t\right)^{2}+\theta_{1} & t_{0} \leq t \leq t_{1} \\ \theta_{1}+\frac{\theta_{1}}{\left(t_{f}-t_{1}\right)}\left(t_{1}-t\right) & t_{1} \leq t \leq t_{f}\end{cases}
$$

where $t_{0}$ is the duration of the SLV vertical flight at the beginning of the trajectory, and $t_{1}$ is time of the first staging, $\theta_{1}$ is the value of the pitch angle at this time, and $t_{f}$ is the burnout (final) time. These three parameters are achieved in the optimization design process.

\section{PROPULSION}

The SLV is powered by ten engines with hydrogen / liquid oxygen fuel, with seven engines in the booster and three engines in the main body, all of which start at the same time as the engine launches, the booster burns until complete discharge and the core burn until insertion (Rostami and Tolouie, 2015):

$$
\begin{gathered}
T^{\prime}=T_{v a c}{ }^{\prime}-p A_{e}{ }^{\prime} \\
T=k T^{\prime} \\
I_{s p}=I_{s p}{ }^{\prime}
\end{gathered}
$$




$$
T_{v a c}=k T_{v a c}{ }^{\prime}
$$

where $A_{e}$ is the exit area, $k=10$ before staging and $k=3$ after staging. The Prime symbol refers to one engine.

\section{AERODYNAMICS}

Aerodynamic forces created a more complete impact in the face of the air particles and the body. One of the important factor in the SLV aerodynamic is the shape of the SLV structure. The Component of producer of the force's and moment are the aerodynamic Coefficients. The drag, lift, and momentum force's is determined by aerodynamic Coefficients and also that force's equation's is given below:

$$
\begin{gathered}
D=q S_{b} C_{D} \\
L=q S_{b} C_{L} \\
M_{A}=q S_{b} l C_{m}
\end{gathered}
$$

In which the aerodynamic coefficients of $C_{D}$ and $C_{L}$ are obtained with respect to the Mach number and the angle of attack variations, given as below:

$$
C_{D}=C_{D}(M, \alpha), C_{L}=C_{L}(M, \alpha)
$$

\section{GRAVITY MODEL}

The Earth is taken to be a rotating, spherical body whose surface is described by the mean sea-level radius $r_{0}$, and whose gravitational acceleration varies with altitude according to the inverse-square law:

$$
g=g_{0}\left(\frac{r_{0}}{r}\right)^{2}
$$

where $\mathrm{r}_{0}=6371 \mathrm{~km}$ and $\mathrm{g}_{0}$ is $9.80665 \mathrm{~m} / \mathrm{s}^{2}$.

\section{OPTIMIZATION DESIGN FORMULATION}

\section{GA OPTIMIZATION}

GA proved their effectiveness as an optimization method for nonlinear problems. Starting with a random initial population of solutions, a GA selects individuals from the population with good chances of reproduction (best objective function) and reproduces the new generation of individuals using operations such as crossover and mutation. The process is repeated several times until it runs a certain number of generations or until a solution considered as optimum is reached.

The parameters of an individual to be optimized are represented by a structure called chromosome and a binary coding is adopted here. For a SLV trajectory design problem each chromosome represents a SLV trajectory and the parameters to be optimized are the trajectory design variables.

The design variables of the problem are the burning time of the first and second stage as well as the pitch angle at the first staging.

The objective function defined to be maximized is the payload Mass criterion, in the presence of final desired parameters of the mission as constraints.

A typical GA flowchart is shown in Fig. 3. 


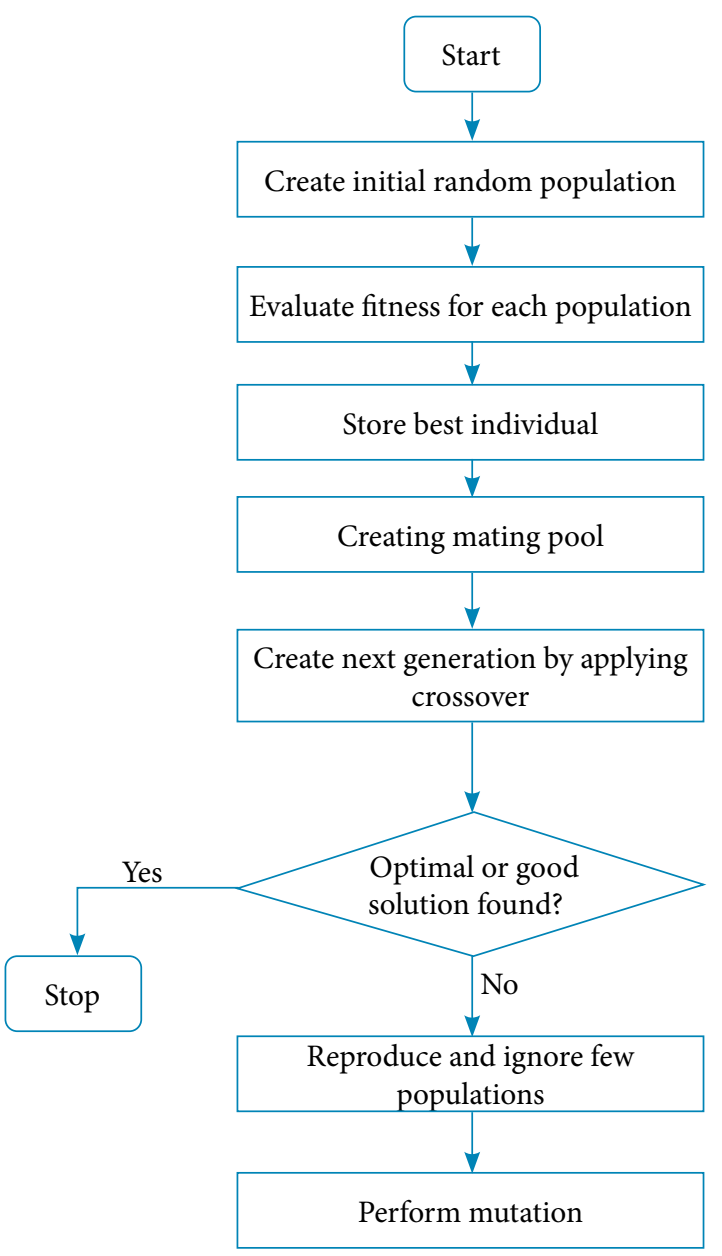

Figure 3. Flowchart of GA (Karsli and Tekinalp, 2005).

The algorithm terminates if the population has converged (does not produce offspring which are significantly different from the previous generation). Then it is said that the genetic algorithm has provided a set of solutions to our problem.

\section{UNCERTAINTY SOURCES}

Regardless of the errors in the model and algorithm related to computer performances, there are several general sources for entering these uncertainties into the simulation. One of the most important of them is the variability of the input values, which have a direct effect on the mass and energy properties. In this work, the SLV aerodynamic coefficients, dry mass and engine thrust are considered with their appropriate error (disturbed) values as uncertainty sources (Table 1). These parameters are assumed to have a normal distribution and the Monte Carlo simulation is used to analyze the uncertainty effect according to Fig. 4.

Table 1. Error value percentage of parameters.

Parameters

Aerodynamic coefficients

Engine Thrust
Percentage of error 


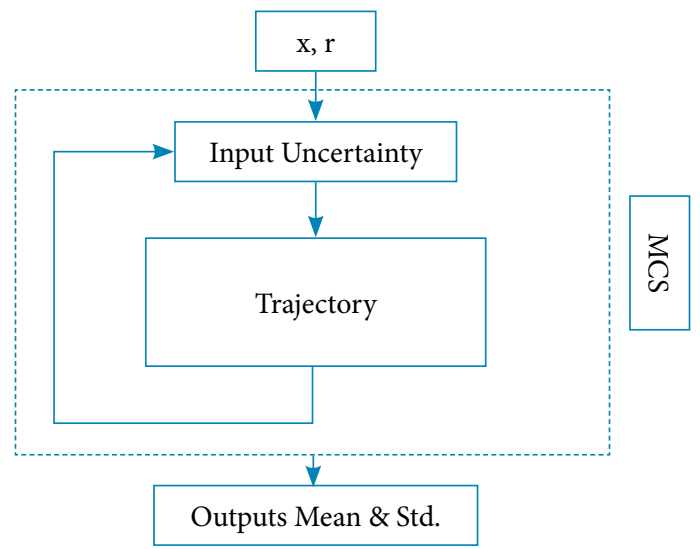

Figure 4. Monte Carlo simulation.

\section{ROBUST OPTIMAL TRAJECTORY FORMULATION IN THE PRESENCE OF UNCERTAINTIES}

Robust design tends to minimize the effect of uncertainty or changes in design parameters on a device without eliminating uncertainty sources. In other words, in a robust optimal design, there is less sensitive to changes in uncontrolled design parameters than the deterministic optimal design.

The main part of a robust design optimization is the objective function definition, which is usually characterized by the mean and variance (or standard deviation) of the uncertain parameters. In general, the mathematical formulation of the robust design optimization is given by the following equation (Mohan, 2002):

$$
\begin{cases}\text { find } & x \\ \min & \tilde{f}(x, p)=F\left(\mu_{f}(x, p), \sigma_{f}(x, p)\right) \\ \text { s.t. } & g(x, p) \leq 0 \\ & x^{L} \leq x \leq x^{U}\end{cases}
$$

where $\mathrm{x}$ and $\mathrm{p}$ can be definite and indefinite design variables, respectively, $\mu_{f}$ and $\sigma_{f}$ are mean and standard deviation of the uncertain parameters. The mean value of the problem in this paper is based on the final altitude of the SLV, as follows:

$$
\mu_{f}=\frac{1}{n} \sum_{i}^{n} h_{i}^{f}
$$

where $\mathrm{n}$ is the number of Monte Carlo executions. The standard deviation of the final height is written as follows:

$$
\sigma_{f}=\sqrt{\frac{1}{n} \sum_{i=1}^{n}\left(h_{i}^{f}-\mu_{f}\right)^{2}}
$$

Finally, minimizing the standard deviation of the final height as the first objective in contrast to maximizing of the payload mass as the second objective are considered. Consequently, the proposed robust optimization formulation is described as a weightedsum multi objective function approach as follows:

$$
\begin{cases}\text { find } & x \\ \min & k_{1} \frac{1}{m_{f}}+k_{2} \sigma_{f} \\ \text { S.t. } & g(x) \leq 0 \\ & x^{L} \leq x \leq x^{U}\end{cases}
$$

where the value of $k_{1}$ is $10^{3}$ and $k_{2}$ is $10^{-4}$, and the design parameter vector $x$ is the vector of the first burning time, the final burning time and of the pitch angle at the first staging, respectively, may be written as below: 


$$
x=\left[\begin{array}{lll}
t_{1} & t_{f} & \theta_{1}
\end{array}\right]
$$

It would be noted that the constraint equations of the optimization problem $g(x)$, are the 3-D equations of motion, and the upper and lower bounds of the vector $x$ is defined later. Briefly, the robust optimization design process of the SLV trajectory is shown in Fig. 5.

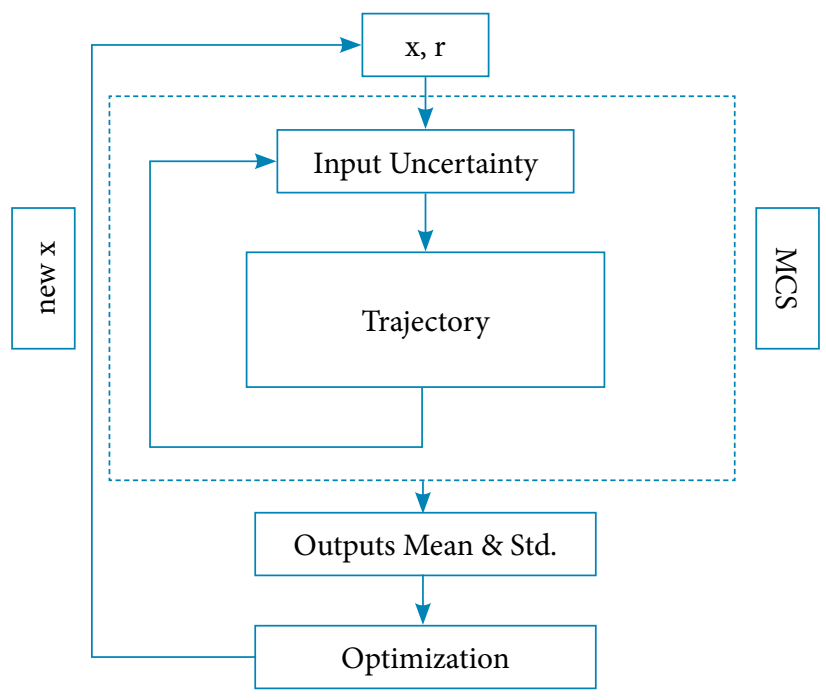

Figure 5. Robust design optimization process.

\section{SIMULATION RESULTS}

To show the effect of uncertainty on the uncertainty analysis results on the optimal trajectory, the uncertainties are introduced in Table 2 and Monte Carlo simulation is performed. The simulation was implemented in MATLAB-R2019b and tested on a PC with $2.5 \mathrm{GHz}$ Intel Core i5 and 4Gbytes RAM running on the 64bit-Windows 10 operating system.

The SLV parameters are presented in table 2:

Table 2. The SLV parameters required for simulation.

\begin{tabular}{|c|c|}
\hline \multicolumn{1}{|c|}{ Parameters } & Value \\
\hline Total weight & $1584542.2(\mathrm{~kg})$ \\
\hline Total Boosters Mass & $756081.136(\mathrm{~kg})$ \\
\hline Engine trust & $2580329.28(\mathrm{~N})$ \\
\hline Main engine burning time & $365.4963(\mathrm{~s})$ \\
\hline Booster burning time & $153.5491(\mathrm{~s})$ \\
\hline Booster Trust & Seven times the engine thrust \\
\hline Isp & $430(\mathrm{~s})$ \\
\hline Longitude & -80.54 \\
\hline latitude & 28.5 \\
\hline Initial height & $0(\mathrm{Km})$ \\
\hline
\end{tabular}


Final desired parameters of the SLV are considered as follows:

$$
\begin{aligned}
h_{f} & =200 \mathrm{Km} \\
V_{f} & =7800 \frac{\mathrm{m}}{\mathrm{s}} \\
\gamma_{f} & =0 \mathrm{deg}
\end{aligned}
$$

The upper bound and lower bound of the vector of decision variable of the optimization problem is as below:

$$
\begin{aligned}
& x^{L}=\left[\begin{array}{lll}
100 & 300 & 20
\end{array}\right]^{T} ; \\
& x^{U}=\left[\begin{array}{lll}
200 & 400 & 30
\end{array}\right]^{T} .
\end{aligned}
$$

and the selected parameters of the GA are:

$$
\begin{aligned}
& N_{\text {Population }}=50 ; \quad N_{\text {Generation }}=100 \\
& P_{\text {Cross }}=0.8 ; \quad P_{\text {Mutation }}=0.05
\end{aligned} .
$$

Figures 6 and 7 show the time history of the SLV altitude and velocity in the presence of uncertainties, respectively, without applying robust optimization. According to Fig. 6, the mean deviation from the final altitude is about $14 \mathrm{~km}$, which requires a great deal of energy to compensate for this altitude. This problem is also seen in Fig. 7 for velocity.

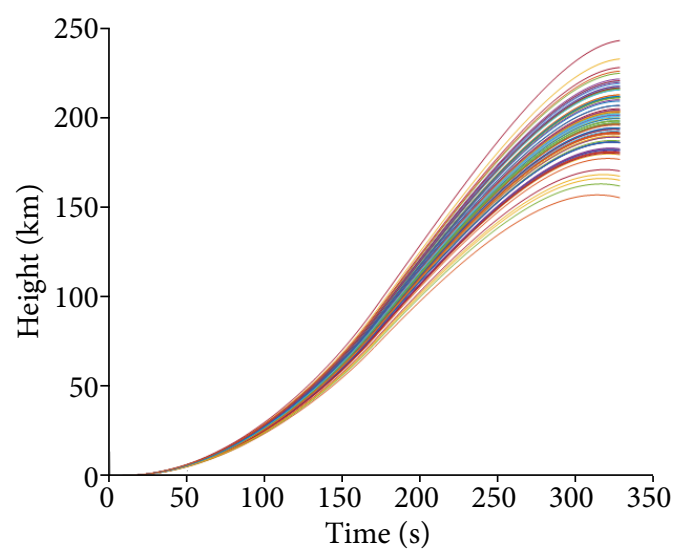

Figure 6. The effect of uncertainties on SLV height with deterministic optimal design.

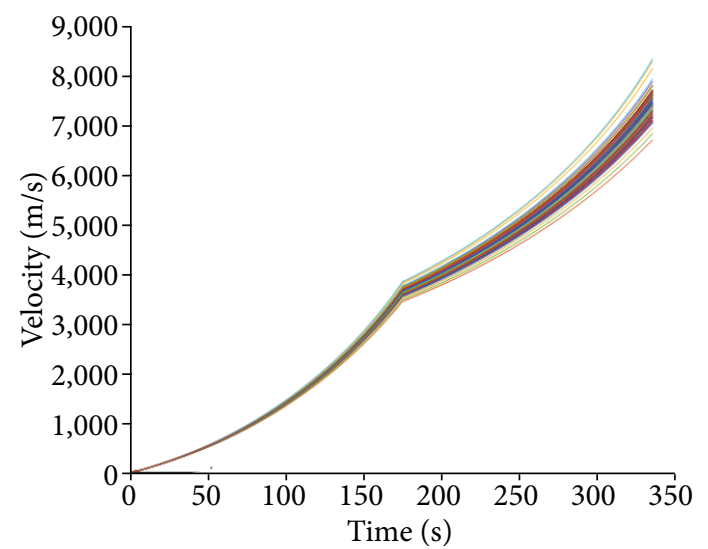

Figure 7. The effect of uncertainties on the SLV final speed with deterministic optimal design. 
Due to applying the robust optimal design of the SLV trajectory as shown in Fig.8, the effect of the uncertainties on the trajectory is significantly reduced. This effect is also shown in Fig. 9 for velocity.

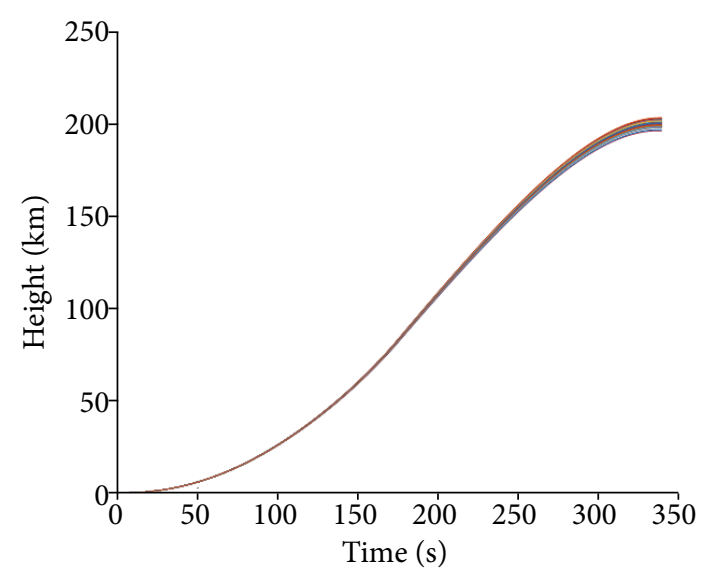

Figure 8. Reducing the effect of uncertainties on SLV height with robust optimal design.

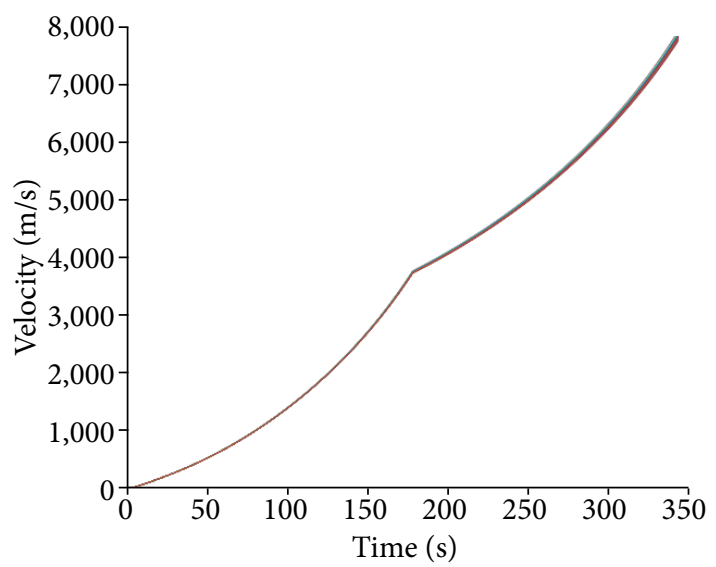

Figure 9. Reducing the effect of uncertainties on SLV velocity with robust optimal design.

By performing a robust design optimization, the mean deviation of the final height is reduced to $1 \mathrm{~km}$, which indicates improved simulation performance in the presence of uncertainties compared to deterministic optimal design. But instead, it results in a penalty in the optimization problem. For example, the final mass in the robust optimization is less than the deterministic optimal and better than basic pitch programming, as shown in Table 3 and Fig. 10.

Figures 11 to 15 also show the comparison of flight parameters for two deterministic optimal and robust optimal approaches.

Table 3. The final parameters in the three modes, the non-optimal, optimal and the robust optimal

\begin{tabular}{|c|c|c|c|}
\hline Parameters & Basic pitch program & Deterministic optimal & Robust optimal \\
\hline $1^{\text {st }}$ stage burning Time $\left(t_{1}\right)$, sec & 153.54 & 143.15 & 170 \\
\hline $2^{\text {nd }}$ stage burning Time $\left(t_{f}\right)$, sec & 365.49 & 379.84 & 330 \\
\hline $1^{s t}$ stage pitch angle $\left(\theta_{1}\right)$, deg & 25.34 & 29.85 & 23.27 \\
\hline Final mass & 162 & 177.5 & 169 \\
\hline
\end{tabular}




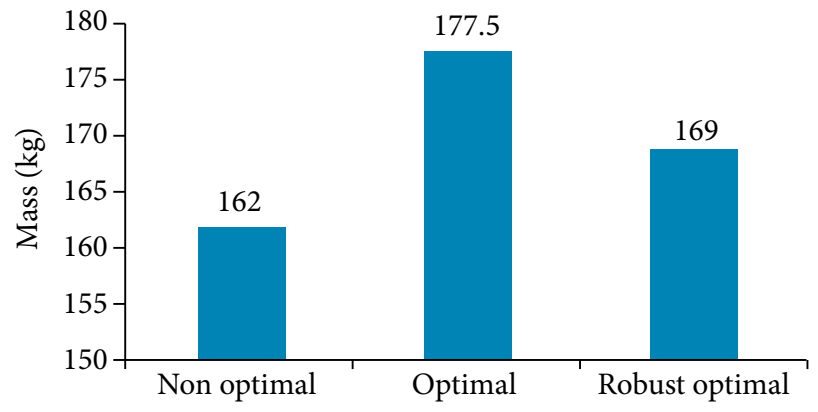

Figure 10. Comparison of the final masses.

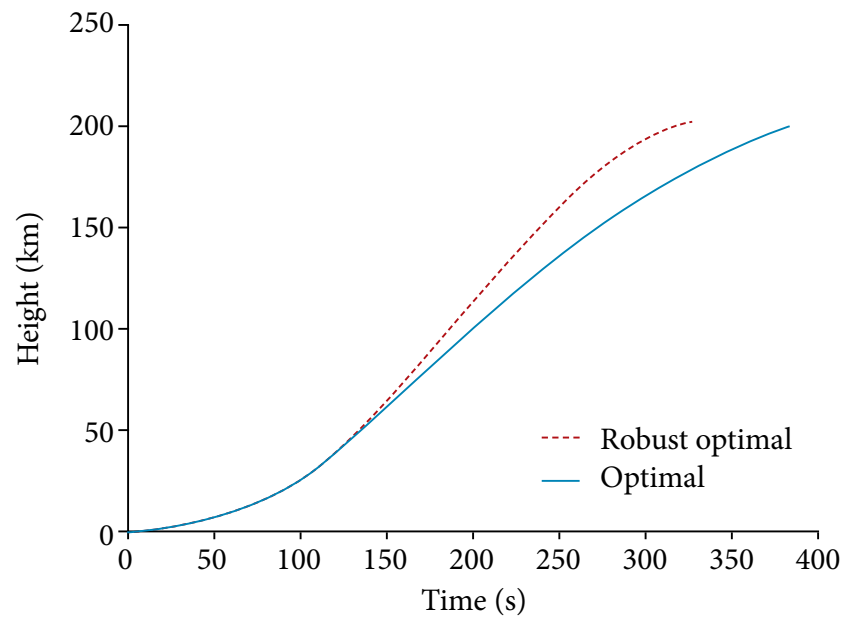

Figure 11. Comparative diagram of the optimum height and the robust optimum height.

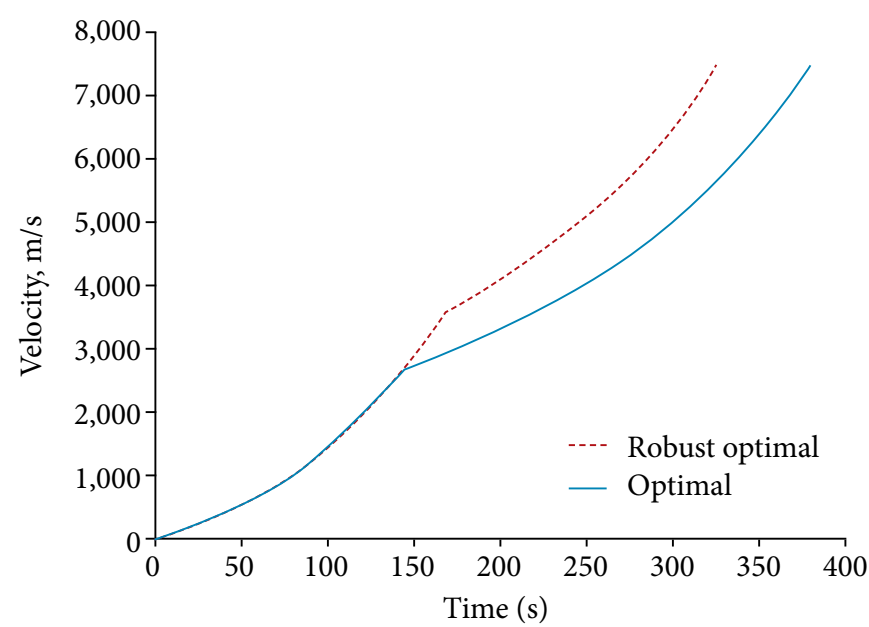

Figure 12. Comparison of speed in optimal and robust optimal. 


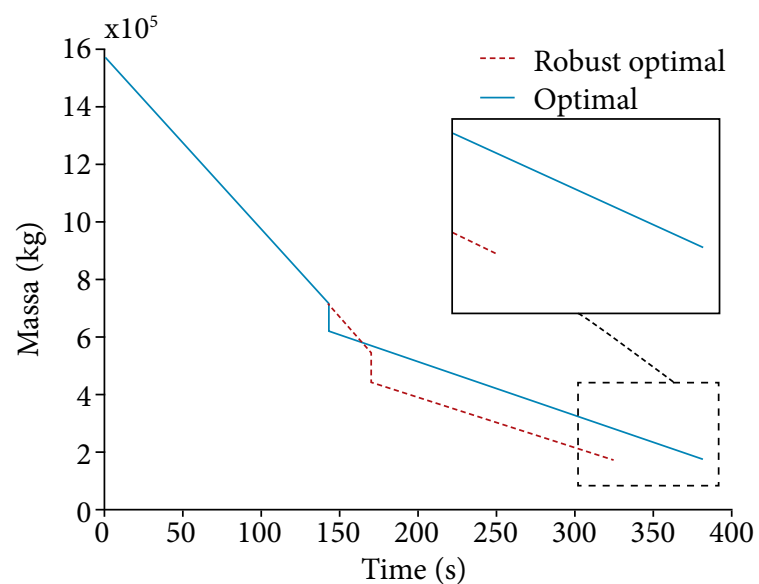

Figure 13. Comparison of mass in optimal and robust optimal.

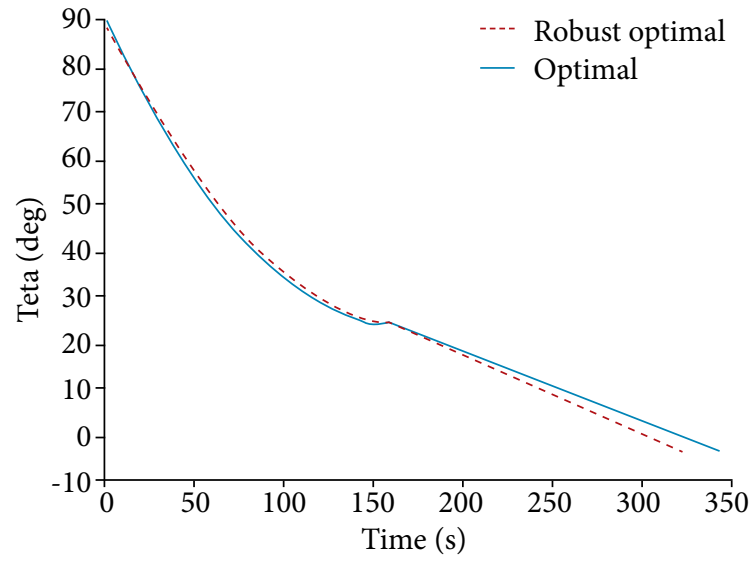

Figure 14. Comparison of pitch angle in optimal and robust optimal.

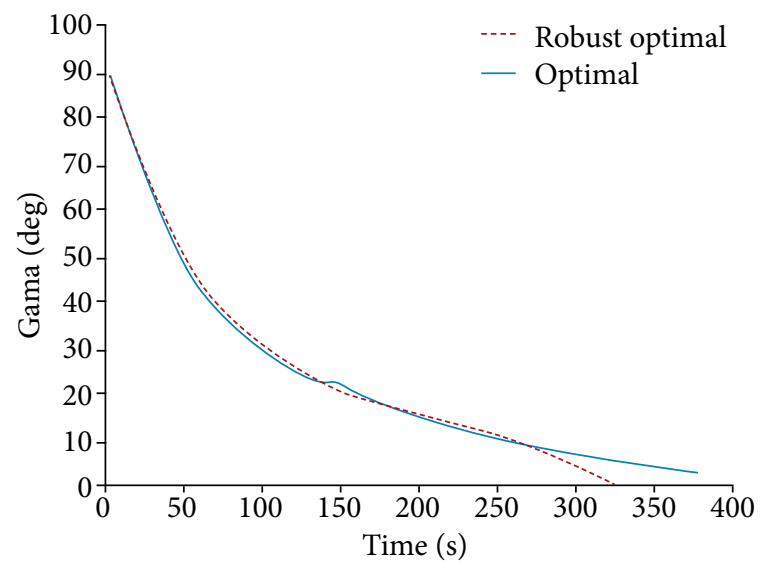

Figure 15. Comparison of trajectory angle in optimal and robust optimal.

\section{CONCLUSION}

The presence of some unknowns in the SLV design or some perturbations along the SLV trajectory causes its performance in real conditions to be different from deterministic computer simulations. Therefore, considering the uncertainties in the design 


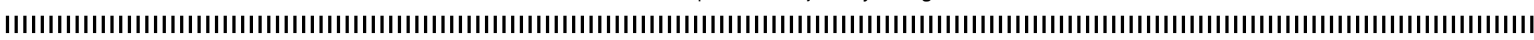

process will increase the success of the mission. Designing a robust trajectory will be an important part of this because it will reduce system control and complexity as well as the order of the actuators. In this work, a GA-based robust optimization is performed to optimize the pitch program to make the SLV more robust to existing uncertainties. The simulation results of the robust design show that the deviation of flight parameters from their desired value is reduced significantly, which indicates improvement of the simulation performance in the presence of uncertainties. It should be noted that in the face of this improvement, there will be penalties in the matter of optimization. For example, the final mass value in the robust optimization is obtained slightly higher than the deterministic optimization, although it is still better than the classical pitch programming method.

\section{AUTHOR'S CONTRIBUTION}

Conceptualization: Zardashti R; Methodology: Zardashti R and Jafari M; Investigation: Hosseeini S M; Simulation: Saadatdar Arani S A; Zardashti R and Hosseeini S M; Writing - Original Draft: Saadatdar Arani S A ;Writing - Review \& Editing: Zardashti $\mathrm{R}$ and Saadatdar Arani S A.

\section{ACKNOWLEDGMENTS}

Editors and authors are thankful to Fundação Conrado Wessel for providing the financial support for publishing this article.

\section{REFERENCES}

Akhtar A, Linshu H (2006) An Efficient Evolutionary Multi-Objective Approach for Robust Design of Multi-Stage Space Launch Vehicle. Paper presented at: 11th AIAA/ISSMO Multidisciplinary Analysis and Optimization Conference. Portsmouth, Virginia, USA. https://doi. org/10.2514/6.2006-7073

Betalebllu AA, Roshanian J, Ebrahimi M (2011). Optimization of Liquid Fuel launch vehicle Design (PhD thesis). Tehran: KN Toosi University of Technology. In Persian.

Grant MJ (2015) Rapid, Robust Trajectory Design Using Indirect Optimization Methods. Paper presented at: AlAA Atmospheric Flight Mechanics Conference. Dalas, TX, USA. https://doi.org/10.2514/6.2015-2401

Hesami Rostami RH, Tolouie A (2015) Designing the Optimal Trajectory of S.A.M in Middle Phase Using Genetic Algorithms and Particle Swarm Optimization (PhD thesis). Tehran: Shahid Behshti University. In Persian.

Hosseini SM, Nosratollahi M, Tolouie (2010) Multidisciplinary design optimization of a launch vehicle. (PhD thesis) Tehran: Shahid Beheshti University. In Persian.

Karsli G, Tekinalp 0 (2005) Trajectory Optimization of Advanced Launch System. Conference present at: Proceedings of 2nd International Conference on Recent Advances in Space Technologies - RAST 2005. Istambul, Turkey. https://doi.org/10.1109/RAST.2005.1512595

Koch PN, Wujek B, Golovidov O (2000) A Multi-Stage, Parallel Implementation of Probabilistic Design Optimization in an MDO Framework. Paper presented at: 8th AIAA/USAF/NASA/ISSMO Symposium on Multidisciplinary Analysis and Optimization. Long Beach, CA, USA.

Liu X, Lu P (2013) Robust Trajectory Optimization for Highly Constrained Rendezvous and Proximity Operations. Paper presented at: AlAA Guidance, Navigation, and Control (GNC) Conference. 2013. p. 4720. https://doi.org/10.2514/6.2013-4720

Luo YZ, Yang (2017) A review of uncertainty propagation in orbital mechanics. Progress in Aerospace Sciences 89: 23-39. https://doi. org/10.1016/j.paerosci.2016.12.002

Marvis DN, Delaurentis DA (2000) Uncertainty Modeling and Management in Multidisciplinary Analysis and Synthesis. Presented at the 38th AIAA Aerospace Sciences Meeting and Exhibit, Reno, NV, USA.

Mohan. S (2002) Robust design (PhD dissertation). Department of Aerospace Engineering, Indian Institute of Technology Bombay.

Okada M, Pekarovskiy A, Buss M (2015) Robust Trajectory Design for Object Throwing based on Sensitivity for Model Uncertainties. Paper presented at: 2015 IEEE International Conference on Robotics and Automation (ICRA): 3089-3094. https://doi.org/10.1109/ICRA.2015.7139623 
Paiva RM, Crawford C, Suleman A (2014) Robust and Reliability-Based Design Optimization Framework for Wing Design. AlAA Journal 52(4): 711-724. https://doi.org/10.2514/1.J052161

Su Z, Wang H (2015) A novel robust hybrid gravitational search algorithm for reusable launch vehicle approach and landing trajectory optimization. Neurocomputing 162(25): 116-127. https://doi.org/10.1016/j.neucom.2015.03.063

Sues R, Cesare M (2000) An innovative framework for Reliability-Base MDO. Conferece presented at: 41st AIAA Structures, Structural Dynamics, and Materials Conference and Exhibit. Atlanta, GA, USA. https://doi.org/10.2514/6.2000-1509

Vinh N (1981) Optimal Trajectories in Atmospheric Flight. New York: Elsevier.

Xue Q, Duan (2017) Robust Attitude Control for Reusable Launch Vehicles Based on Fractional Calculus and Pigeon-inspired Optimization. IEEE/CAA Journal of Automatica Sinica, 4(1): 89-97. https://doi.org/10.1109/JAS.2017.7510334

Yao W, Chen X, Luo W, Tooren M, Jian Guo (2011) Review of uncertainty-based multidisciplinary design optimization methods for aerospace vehicles. Progress in Aerospace Sciences 47(6): 450-479. https://doi.org/10.1016/j.paerosci.2011.05.001

Wazed MA, Shamsuddin A, Nukman Yusoff (2009) Uncertainty Factors in Real Manufacturing environment. Australian Journal of Basic and Applied Sciences, 03 (2): 342- 351.

Zaman K, McDonald M, Mahadevan S, Green L (2009) Robustness-based design optimization under data uncertainty. Structural and Multidisciplinary Optimization 44(2):183-197. https://doi.org/10.1007/s00158-011-0622-2

Zipfel PH (2007) Modeling and Simulation of Aerospace Vehicle Dynamics-American Institute of Aeronautics and Astronautics. Reston, VA: American Institute of Aeronautics and Astronautic. https://doi.org/10.2514/4.862182 